\title{
Does conventional abdominal preoperative ultrasonography have a role in the detection of pancreatic insulinoma? A case report
}

\author{
L. Spadaro, O. Magliocco, G. Trovato, F. Purrello
}

Dpt di Medicina Interna e Medicina Specialistica, Università degli Studi di Catania, Ospedale Garibaldi, Italy; fpurrell@unict.it

Received 2 September 2009; revised 14 October 2009; accepted 16 October 2009.

\begin{abstract}
Combinations of strategies as MRI, endoscopic sonography, selective arteriography, were the first choice for the detection of pancreatic insulinoma. In these proposal, therefore, abdominal was not in- cluded at all. Case presentation: a 78-year-old no diabetic women was referred to us because fasting hypoglycemic symptoms. The clinical and laboratory findings suggested an insulinoma. Abdominal ultrasound showed a small solid mass in the head-istmus pancreatic tract. Conclusion: the solid mass was confirmed with a contrast-enhanced- computed tomography of the abdomen. A surgical enucleation of the tumor was achieved by laparoscopy and histological examination of the specimen established a diagnosis of insulinoma. This case reinforced the value of the conventional transabdomen ultrasound in addition to accuracy of anamneses and biochemical tests as the first step in the hospital clinical setting for management of pancreatic insulinoma, reserving as the second step more expensive and invasiveness techniques.
\end{abstract}

Keywords: Lunch Time Napping; Mental Work Efficiency

Insulinomas are rare endocrine tumors of the pancreas. Their incidence was estimated to be four cases per 1 million person-years [1]. In the earliest stages of the tumor's clinical history, they usually manifest because of hypersecretion of insulin, responsible for the typical hypoglycemic symptoms. The three criteria (Whipple's triad or Whipple's criteria) on which hypoglycemia due to pancreatic islet-cell disease (most commonly insulinoma) is diagnosed are: 1) symptoms known or likely to be caused by hypoglycemia, 2) low blood glucose levels associated with clinical signs, and 3 ) reversal of clinical signs with the administration of glucose. The optimal therapy is curative surgical removal of the tumor. Therefore, preoperative localization of the lesion is crucial for determining the proper surgical treatment. However, in several cases, insulinoma identification remains extremely difficult and there was a continuing debate on the optimal strategy for his localization. Some authors have proposed MR as the first choice for preoperative imaging $[2,3]$, whereas recent reports suggested a combined imaging protocol that consists of both dual-phase thin-section multi-detector CT and endoscopic sonography $[4,5]$. Actually, conventional abdominal ultrasonography was not included in the imaging protocol due to his limited successful reports. We wish to present our own experience with abdominal conventional ultrasound in a case of insulinoma.

A non-diabetic 78-year-old woman was brought to our attention because of hypoglycaemic symptoms. The patient had a history of mild and transient sweating, anxiety and trembling recurring for about 2 years during her activities of ordinary life. These symptoms usually disappeared after food ingestion. Ten days before her referral to our hospital, she had a more severe episode with sweating, trembling, anxiety, palpitations, nausea, dizziness with tiredness and a severe headache crisis. She had been seen by her family physician. The practitioner had ordered general blood tests that had revealed low serum glucose level $(2.2 \mathrm{mmol} / \mathrm{L}, 40 \mathrm{mg} / \mathrm{dL})$. Therefore, he suggested the admission to the hospital, to determine the cause of hypoglycaemia.

She had a history of hypertension and dyslipidemia on pharmacological treatment. She didn't have any treatment or drugs that might have induced hypoglycaemia. On admission to our hospital, on examination, the patient was overweight; her height was $165 \mathrm{~cm}$ and body weight 94,5 Kg, BMI 34,74, CV $118 \mathrm{~cm}$. Blood pressure was $140 / 80 \mathrm{mmHg}$ and the pulse was 68 . Neurological examination and an electrocardiogram were normal. Laboratory tests were performed: urinalysis, blood cell counts, and biochemical tests (e.g. the liver and kidney function tests) other than plasma glucose concentration 
were unremarkable.

On the second hospital day, we performed the supervised 72 -Hour Fast. At the $12^{\text {th }}$ hour, the patient showed pallor, sweating, trembling, tiredness, nervousness and confusion. The plasma glucose level was $42 \mathrm{mg} / \mathrm{dl}$. Therefore, we stopped the test through correction of the low glucose level by food ingestion with rapid relieving of the symptoms ("Whipple's triad") (Table 1). Plasma insulin levels during the test were also shown in Table 1. These data supported the diagnostic suspect of insulinoma.

As a first attempt to locate it, an abdominal ultrasonography of the pancreas was performed with a $3.5 \mathrm{MHz}$ probe mechanical real time sector scanner (Logiq 500). Patient was examined in a semierect position after fasting for 12 hours. Scans of the head, body, and tail of the pancreas were obtained in both transverse and longitudinal planes without respiratory maneuvers, by using the fluid-filled stomach as an acoustic window. The examination showed a 12,4 $\mathrm{mm} \times 13,7 \mathrm{~mm}$ hypoechoic solid mass, with the echo texture finer in relation to surrounding pancreatic tissue, localized in the head-isthmus pancreatic tract, without dilatation of the Wirsung's duct. (Figure 1, left). A contrast-enhanced computed tomography of the abdomen was then performed. The patient was scanned in suspended respiration, following the oral administration of contrast material to visualize the small bowel. Pre-contrast abdominal scans CT, revealed the presence of a protrusion of the pancreatic contour in the head-isthmus pancreatic tract, but itself is non-specific. After the bolus injection of contrast material and rapid sequential scanning, a transient increase in contrast enhancement (tumor blush) in the surrounding pancreatic tissue was observed, confirming an area of increased density of 1,5 cm (Figure 1, right). One month later, a surgical enucleation of the tumor was achieved by
Table 1. 72-Hour fast (date onset of the fast was the last ingestion of calories, 12 hours before the first measurement; all of the non essential medications were stopped; no assumption of caffeine or tobaccos).

\begin{tabular}{ccrrr}
\hline Time & $\begin{array}{c}\text { Symptoms } \\
\text { or signs }\end{array}$ & Glucose & Insulin & c-peptide \\
\hline $01: 00$ & none & 48 & 15 & 1,79 \\
p.m. & & $\mathrm{mg} / \mathrm{dl}$ & $\mu \mathrm{U} / \mathrm{mL}$ & $\mathrm{nmol} / \mathrm{L}$ \\
03:00 & none & 59 & 11 & 1,29 \\
p.m. & & $\mathrm{mg} / \mathrm{dl}$ & $\mu \mathrm{U} / \mathrm{mL}$ & $\mathrm{nmol} / \mathrm{L}$ \\
$05: 00$ & none & 64 & 10 & 1,40 \\
p.m. & & $\mathrm{mg} / \mathrm{dl}$ & $\mu \mathrm{U} / \mathrm{mL}$ & $\mathrm{nmol} / \mathrm{L}$ \\
11:00 & none & 62 & 4 & 0,89 \\
p.m. & & $\mathrm{mg} / \mathrm{dl}$ & $\mu \mathrm{U} / \mathrm{mL}$ & $\mathrm{nmol} / \mathrm{L}$ \\
$05: 00$ & none & 69 & 10 & 1,35 \\
a.m & & $\mathrm{mg} / \mathrm{dl}$ & $\mu \mathrm{U} / \mathrm{mL}$ & $\mathrm{nmol} / \mathrm{L}$ \\
11:00 & none & 53 & 12 & 1,13 \\
a.m. & & $\mathrm{mg} / \mathrm{dl}$ & $\mu \mathrm{U} / \mathrm{mL}$ & $\mathrm{nmol} / \mathrm{L}$ \\
12:00 & Whipple's triad: & 42 & 7 & 1,04 \\
a.m. & end of the fast & $\mathrm{mg} / \mathrm{dl}$ & $\mu \mathrm{U} / \mathrm{mL}$ & $\mathrm{nmol} / \mathrm{L}$ \\
\hline
\end{tabular}

laparoscopy, which was performed initially to confirm by direct view the presence of the small nodule on the surface of the pancreatic; its enucleation was then carried out. Histological examination of the specimen established a diagnosis of an endocrine tumour of the pancreas compatible with an insulinoma, confirmed by the presence of insulin secretory granules. The patient's clinical symptoms completely disappeared.

Endocrine tumors of the pancreas originate from multipotential stem cells that have retained the capacity to proliferate and differentiate themselves in the various cellular lines that make up this group of neoplasms. Insulinomas represent the most frequently found functioning endocrine tumors of the pancreas and, in most cases, are benign (85\%-99\%), single (93\%-98\%) and less than
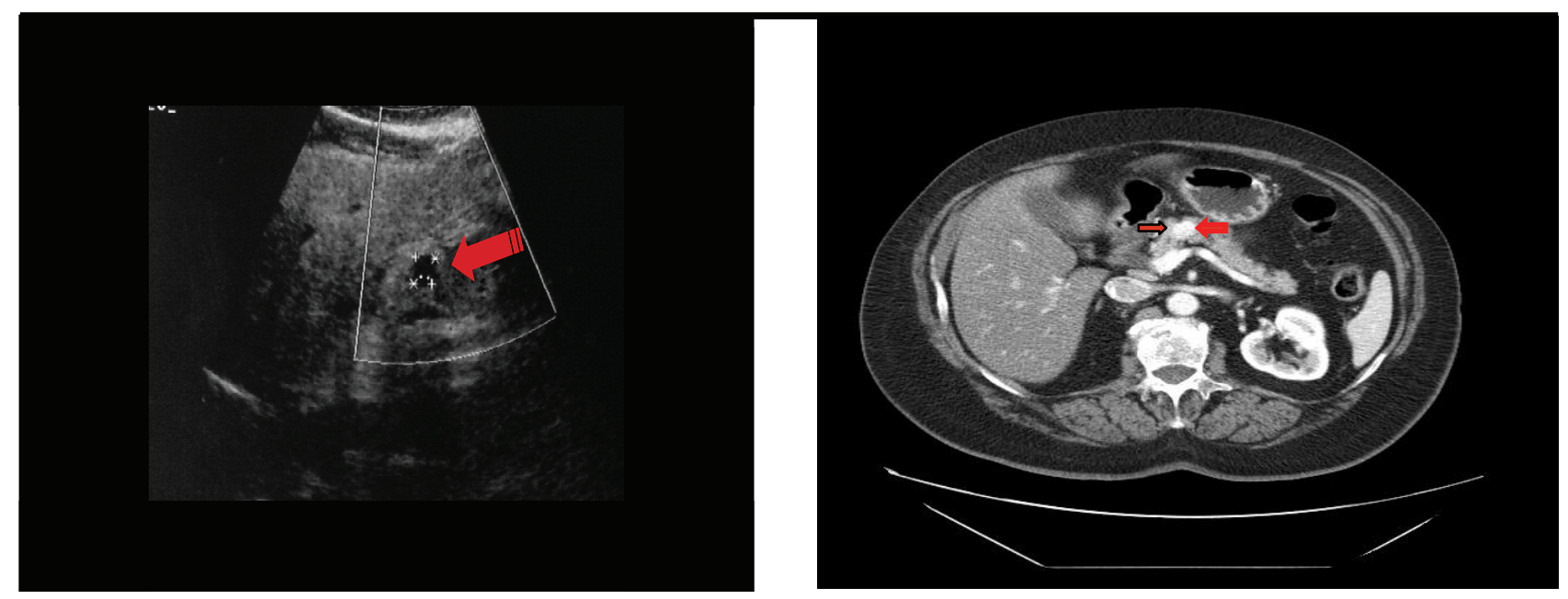

Figure 1. Imaging studies of the pancreatic lesion. Ultrasound (left) and CT (right) technique show the characteristic mass of the pancreas (arrows). 
$2.5 \mathrm{~cm}$ in diameter [6,7]. Age of onset is usually $40-60$ years, ranging from 6 weeks to 70 years. No sex difference has been reported [8]. In our case, the patient was a 78-year-old female.

Insulinoma become clinically evident because of signs and symptoms linked to hypoglycemia due to the hypersecretion of insulin. The symptoms of hypoglycemia arise from the autonomic nervous system and from an insufficient supply of glucose to the brain (neuroglycopenia). During acute insulin-induced hypoglycemia in healthy persons, symptoms have been recognized at plasma glucose levels of approximately $60 \mathrm{mg}$ per deciliter as measured in arterialized venous blood, and impairment of brain function has occurred at approximately $50 \mathrm{mg}$ per deciliter [9-10-11]. In our patient, the first report of low plasma glucose levels $(40 \mathrm{mg} / \mathrm{dL})$ was associated with typical hypoglycemic symptoms, sweating, trembling, anxiety, palpitations, nausea, dizziness with tiredness and headache crisis. Moreover, her past history was positive for sporadic and vague symptoms, recurring for about 2 years, but no plasma glucose levels was measured before. It has been reported that the time interval from the onset of symptoms to diagnosis ranges from 10 days to 30 years (median 2 years), and that the hypoglycemic episodes may happen at irregular intervals with a varying duration [8].

The symptoms intermittency and the multifaceted characters delay the diagnosis, although all signs and symptoms are usually reverted rapidly by oral or parenteral glucose administration. Obesity or weight gain is present in $25 \%$ of patients, justified, at least in part, by the need for frequent feeding. Accordingly, our patient was overweight, and she reported a weight gain (over 20 $\mathrm{kg}$ ) in the past two years. Because of the lack of specificity of the symptoms, it is necessary to measure a low glucose level at the time that the spontaneous symptoms occur and that the symptoms are relieved through correction of the low glucose level ("Whipple's triad") before concluding that the patient have a hypoglycemic disorder. The supervised 72-hour fast is the classic diagnostic test for hypoglycemia. In our case, the supervised 72-hour fast confirmed a hypoglycemic disorder. In addition, the appearance of the Whipple's triad associated with plasma glucose level $\leq 45 \mathrm{mg} / \mathrm{dl}$, insulin level $\geq 6 \mu / \mathrm{mL}$ and c-peptide level $\geq 0.2 \mathrm{mmol} / \mathrm{L}$ suggested the diagnosis of insulinoma [12].

When the diagnosis of pancreatic insulinoma has been established clinically and biochemically, the precise localization of the tumor is crucial, to facilitate the surgical resection and to prevent a blind partial or subtotal pancreatectomy [13]. Insulinomas are usually small and difficult to detect. The main problem in detecting a pancreatic insulinoma lies in the organ's anatomic structure and the tumor's small dimensions at clinical appearance. Most of the tumors are in the body-tail area of the pan- creas and are less than $2 \mathrm{~cm}$ in diameter [14]. Many procedures have been proposed for their localization. These imaging techniques vary in accuracy, invasiveness, operator dependence, and cost. Recent protocols propose the combination of advanced imaging techniques as well as MRI, endoscopic sonography, and CT as the first choice for preoperative detection of insulinomas [2,4]. In these proposals, therefore, abdominal ultrasonography is not included at all. However, ultrasonography is a noninvasive and relatively inexpensive technique, and actually it is easily available everywhere. In the case report we discuss here, we performed abdominal ultrasonography as the imaging technique of first choice because it was immediately available in our unit. In our patient, this technique correctly localized a pancreatic solid mass as a well-defined, hypoechoic lesion, without calcifications or necrosis. This finding was then confirmed by CT examination.

Early studies of preoperative ultrasound localization primarily used static scanners, yielding disappointing results. More recently, a higher sensitivity (approximately $60-80 \%$ ) has been reported using real-time US equipment $[7,15,16]$. This level of accuracy was highly dependent on meticulous attention to scanning technique [6]. Ultrasonographic detection of insulinomas was difficult because of the frequent location at the body-tail area of the pancreas and the small sizes of the tumours in generally obese patients. In our patient insulinoma was small, $<1.5 \mathrm{~cm}$, and was localized in head-isthmus pancreatis tract. It is important to emphasize the improvement in ultrasonographic technology in terms of spatial resolution with increased diagnostic sensitivity and accuracy. In addition, ultrasonography technique enabled us to view continuous images of the lesion from several angles, with relative ease compared to other imaging technique.

In the current case, after correct diagnosis and localization of pancreatic insulinoma, surgical resection was performed successfully and without complication. Actually, after one year, the patient was free from symptoms.

In conclusion, the present case supported the value of the conventional abdomen ultrasonography as the first step in the preoperative localization of pancreatic insulinomas, reserving as the second step more expensive and invasiveness techniques.

\section{REFERENCES}

[1] Service, F.J., McMahon, M.M., O’Brien, P.C., et al. (1991) Functioning insulinoma: incidence, recurrence, and long term survival of patients: a 60-years study. Mayo Clin Proc, 66, 711-719.

[2] Virally, M.L., Guillausseau, P.J. (1999) Hypoglycemia in adults. Diabetes e Metabolism, 25, 477-490. 
[3] Catalano, C., Pavone, P., Laghi, A., et al. (1999) Localization of pancreatic insulinomas with MR imaging at 0.5 T. Acta Radiol, 39, 644-648.

[4] Moore, N.R., Rogers, C.E., Britton, B.J. (1995) Magnetic resonance imaging of endocrine tumors of the pancreas. Br J Radiol, 68, 341-347.

[5] Gouya, H., Vignaux, O., Augui, J., Dousset, B., Palazzo, L., Louvel, A., Chaussade, S., Legmann, P. (2003) CT, endoscopic sonography, and a combined protocol for preoperative evaluation of pancreatic insulinomas. AJR, 181, 987-992.

[6] Schumacher, B., Lubke, H.J., Frieling, T., Strohmeyer, G., Starke, A.A. (1996) Prospective study on the detection of insulinomas by endoscopic ultrasonography. Endoscopy, 28, 273-276.

[7] Liu, T.H., Tseng, H.C., Zhu, Y., Zhong, S.X., Chen, J., Cui, Q.C. (1985) Insulinoma: an immunohistochemical and morphologic analysis of 95 cases. Cancer, 56, 1420-1429.

[8] Stefanini, P., Carboni, M., Patrassi, N., Basoli, A. (1974) Beta islet-cell tumors of the pancreas: result of a study on 1067 cases. Surgery, 75, 597-609.

[9] Villary, M.L., Guillausseau, P.J. (1999) Review: Hypoglycemia in adults. Diabetes \& Metabolism, 25, 477-490.

[10] Schwartz, N.S., Clutter, W.E., Shah, S.D., Cryer, P.E. (1987) Glycemic thresholds for activation of glucose counterregulatory systems are highter than the threshold for symptoms. J Clin Invest, 79, 777-781.

[11] Mitrakou, A., Ryan, C., Veneman, T., et al. (1991) Hier- rarchy of glycemic thresholds counterregulatory hormone secretion, symptoms, and cerebral dysfunction. Am J Physiol, 260, E67-E74.

[12] Cyper, P.E. (1993) Glucose counterregulation: the physiological mechanisms that prevent or correct hypoglycaemia. In: Frier, B.M., Fisher, B.M., (eds.), Hypoglycaemia and Diabetes: Clinical and Physiological Aspects. Edward Arnold, London, 34-55.

[13] Service, F.J. (1995) Review: Hypoglycemic disorders.. NEJM, 332, 1144-1152.

[14] Placzkowski, K.A., Vella, A., Thompson, G.B., Grant, C.S., Reading, C.C., Charboneau, J.W., Andrews, J.C., Lloyd, R.V., Service, F.J. (2009) Secular trends in the presentation an management of functioning insulinoma at the majp clinic, 1987-2007. J Clin Endocrinol Metab.

[15] D’Onofrio, M., Mansueto, G., Vasori, S., Falconi, M., Procacci, C. (2003) Contrast-enhanced ultrasonographic detection of small pancreatic insulinoma. $J$ Ultrasound Med, 22, 413- 417 .

[16] Angeli, E., vanzulli, A., Castrucci, M., Venturini, M., Sironi, S., Zerbi, A., Di Carlo, V., Del Maschio, A. (1997) Value of abdominal sonography and MR imaging at $0.5 \mathrm{~T}$ in preoperative detection of pancreatic insulinoma: a comparison with dynamic CT and angiography. Abdom Imaging, 22(3), 295-303.

[17] Galiber, A.K., Reading, C.C., Charboneau, J.W., Sheedy, P.F., James, E.M., Gorman, B., Grant, C.S., van Heerden, J.A., Telander, R.L. (1998) Localization of pancreatic insulinoma: comparison of pre-and intraoperative US with CT and angiography. Radiology, 166(2), 405-8. 